\title{
Prediction Model of the Fusion Microzone on Sliding Contact Surface
}

\author{
Yan Lu and Zuomin Liu \\ Institute of Tribology, Wuhan University of Technology, Wuhan 430070, China \\ Correspondence should be addressed to Zuomin Liu, liuzm98@263.net
}

Received 12 August 2011; Accepted 5 September 2011

Academic Editor: T. Basak

Copyright ( $) 2011$ Y. Lu and Z. Liu. This is an open access article distributed under the Creative Commons Attribution License, which permits unrestricted use, distribution, and reproduction in any medium, provided the original work is properly cited.

The current paper is motivated by the need to understand the factors in generating the fusion microzone in sliding systems. The objectives are to analyze the different elements' varied influence on the engineering surface's temperature rise. The current paper developed the prediction model based on the thermal conduct theory. A solution based on the Green's function method is combined with the grid method for calculating the temperature rise and distribution. The research indicates that: frictional heat is closely related to the sliding velocity, its value is in proportion to the sliding velocity; the thermal properties of the material are one of the key points to decide the temperature rise; the load is another main factor in increasing the temperature rise; comparing with other elements, the roughness may be the least effective to the temperature rise.

\section{Introduction}

In the sliding contact process, a temperature variation in the contacting bodies that resulted from frictional heating will cause many related engineering problems, such as wear, fusion, and deformation. Since Blok [1] and Jaeger and Carslaw [2] pioneered the studies of temperature rise at the contact surfaces due to moving heat surfaces, there are more researches aimed at studying the temperature rise and distribution. Vick and Furey [3] deduced the base theory of the frictional heat and calculated a set of regularly arranged contact areas' temperature distribution; Chen et al. [4] used the classic theory to calculate the local temperature rise that greatly influences the glue, wear, and local plastic deformation. Chen and $\mathrm{Li}$ [5] established a wear model to simulate sliding processes in order to analyze the frictional heating. Bogdanovich and Tkachuk [6] presented some experiments to understand thermal phenomena in a sliding contact, revealing that asperities in contact were found to undergo the pulse effect of the temperature approaching the melting point of one of the friction members. But few researches are focused on the temperature distribution of the realistic engineering surface which was constructed by a set of patches whose sizes and shapes are irregularly. The major objective of the present paper is to study the real contact area's frictional heat. The developed sliding thermal model can deduce the equation of thermal conduction. The solution is used to demonstrate the effects of the contact area's roughness, the parameters of materials, velocity of the sliding, and the ability of load.

\section{Mathematic Model of Friction Heat}

When the true engineering surfaces slide over each other, the frictional heat is generated by the contact area which is a set of discontinuous asperities with different sizes, varied shapes. Then the system of heat generation on frictional surfaces can be modeled as finite heat sources moving on half-infinite homogeneous body [7-9], as shown in Figure 1. It consists of two contacting semi-infinite regions, the rough surface with normal load $F$ and the flat one move with the sliding velocity $V$ in the $y$-direction and the frictional thermal will disappear to these two bodies (without considering the consumption of energy).

It can be seen clearly from the Figure 1 that the frictional heat is generated by part of the hole. It is the main factor in generating the fusion point. As the test shows that when M50 $(\mathrm{Ra}=0.24-0.38 \mu \mathrm{m})$ sliding under the load $F=10 \mathrm{~N}$, test 


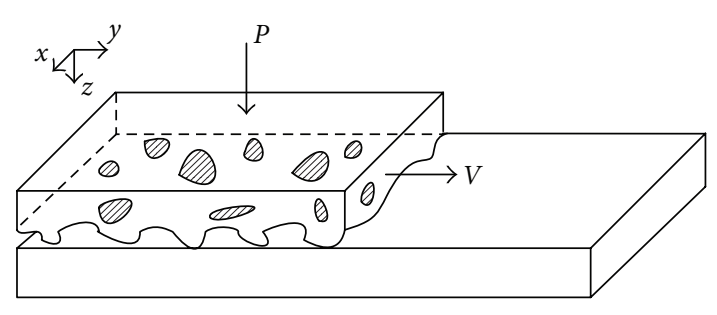

FIgURE 1: Theory figure of the rough surface's sliding.

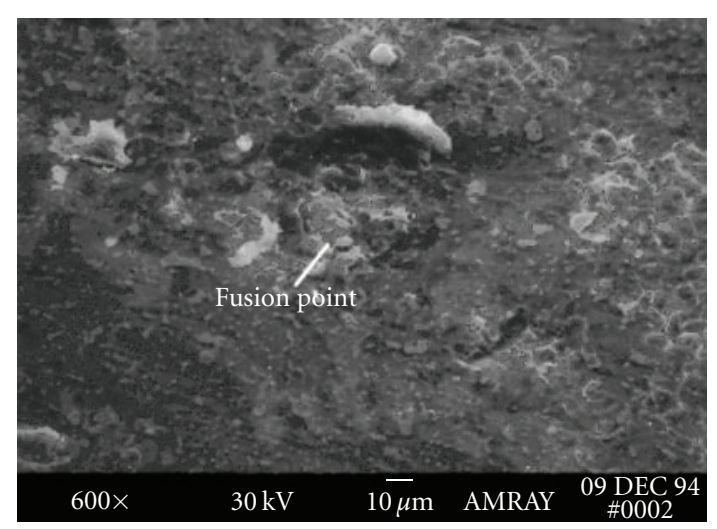

FIGURE 2: Fusion micro-zone on the M50 engineering surface.

temperature $T=400^{\circ} \mathrm{C}$, with $V=1 \mathrm{~m} / \mathrm{s}$, the fusion points are generated (Figure 2).

2.1. Thermal Equation of the Contact Region. According to the theory of the thermal conduction:

$$
\text { heat } \begin{aligned}
t_{2}-\text { heat } t_{1}= & \text { the boundary heat inflow } \\
& + \text { heat generated by the heat source. }
\end{aligned}
$$

Set $u(x, y, z, t)$ to represent the temperature in which the body moves to the position $(x, y, z)$ at time $t, C$ is the specific heat capacity, $V$ is the relative velocity, $\rho$ is density, and $k$ is the coefficient of heat conducting, then

$$
\begin{gathered}
\iiint_{D} C \rho\left[u\left(x, y, z, t_{2}\right)-u\left(x, y, z, t_{1}\right)\right] d x d y d z \\
=\int_{t_{1}}^{t_{2}} d t \iint_{\partial D} k \frac{\partial u}{\partial n} d S \\
\Longrightarrow \int_{t_{1}}^{t_{2}} d t \iiint_{D} C \rho \frac{\partial u}{\partial t} d x d y d z \\
\quad+\int_{t_{1}}^{t_{2}} d t \iiint_{D} C \rho \frac{\partial u}{\partial y} \frac{\partial y}{\partial t} d x d y d z \\
=\int_{t_{1}}^{t_{2}} d t \iiint_{D} \nabla \cdot(k \nabla u) \\
\Longrightarrow C \rho \frac{\partial u}{\partial t}+C \rho \cdot V \cdot \frac{\partial u}{\partial y}=\nabla \cdot(k \nabla u)
\end{gathered}
$$

$$
\begin{aligned}
& \Longrightarrow \frac{\partial u}{\partial t}+V \cdot \frac{\partial u}{\partial y}=a^{2}\left(\frac{\partial^{2} u}{\partial x^{2}}+\frac{\partial^{2} u}{\partial y^{2}}+\frac{\partial^{2} u}{\partial z^{2}}\right) \\
& \Longrightarrow u_{t}=a^{2} \Delta u-V \cdot u_{y} .
\end{aligned}
$$

Here, $\Delta=\partial^{2} / \partial x^{2}+\partial^{2} / \partial y^{2}+\partial^{2} / \partial z^{2}, a^{2}=k / C \rho$ is heat diffusion coefficient.

Here the thermal boundary is the second boundary condition:

$$
k \frac{\partial u}{\partial n}= \begin{cases}q, & \text { contact region }(z=0) \\ 0, & \text { uncontact region }(z \neq 0) .\end{cases}
$$

Here $q$ is the heat density generated by the sliding contact region's frictional heat, it can be demonstrated by

$$
q=\mu \frac{F}{\sum_{i=1}^{s u m} A_{i}} V
$$

Here $\mu$ is friction coefficient; $F$ is pressure of union area; $V$ is sliding velocity; $\sum_{i=1}^{\mathrm{sum}} A_{i}$ is efficient contact area ( $i$ is the number of the contact area). Assume that there is no energy loss during the sliding process. Initial condition at the beginning of the sliding $t=0$,

$$
u(x, y, z, t)=T_{0} .
$$

2.2. Solving the Thermal Control Equation. The frictional heat control equation of the sliding contact region is constructed by (2), (3), and (5), then according to Green's method of the partial differential equation (3), $d u=$ $q \iint_{A_{i}}[G]_{z=0} d x^{\prime} d y^{\prime}$, the Green Function can be presented as

$$
\begin{gathered}
G=\frac{1}{\left[4 a \sqrt{\pi\left(t-t^{\prime}\right)}\right]^{3}} . \\
\exp \left\{-\frac{\left(x-x^{\prime}\right)+\left[\left(y-y^{\prime}\right)-V\left(t-t^{\prime}\right)\right]^{2}}{4 a^{2}\left(t-t^{\prime}\right)}\right\} .
\end{gathered}
$$

According to it, the influence coefficient of the temperature rise can be solved:

$$
T=\int_{t^{\prime}=0}^{t}\left(\iint_{A_{i}}[G]_{z=0} d x^{\prime} d y^{\prime}\right) d t^{\prime} .
$$

The contact patch is defined as the rectangular region, $x$ direction from $i-1 / 2$ to $i+1 / 2, y$-direction from $j-1 / 2$ to $j+1 / 2$, then the thermal influent coefficient can be presented as

$$
\begin{gathered}
T=\frac{1}{4 \sqrt{\pi}} \int_{t^{\prime}=0}^{t} T_{x} \cdot T_{y} d t^{\prime}, \\
T_{x}=\left\{\operatorname{erf}\left[\frac{x_{a}-x_{i-1 / 2}}{4 a^{2}\left(t-t^{\prime}\right)}\right]-\operatorname{erf}\left[\frac{x_{a}-x_{i+1 / 2}}{4 a^{2}\left(t-t^{\prime}\right)}\right]\right\}, \\
T_{y}=\left\{\operatorname{erf}\left[\frac{y_{b}-y_{j-1 / 2}-V\left(t-t^{\prime}\right)}{4 a^{2}\left(t-t^{\prime}\right)}\right]\right. \\
\left.-\operatorname{erf}\left[\frac{y_{b}-y_{j+1 / 2}-V\left(t-t^{\prime}\right)}{4 a^{2}\left(t-t^{\prime}\right)}\right]\right\} .
\end{gathered}
$$




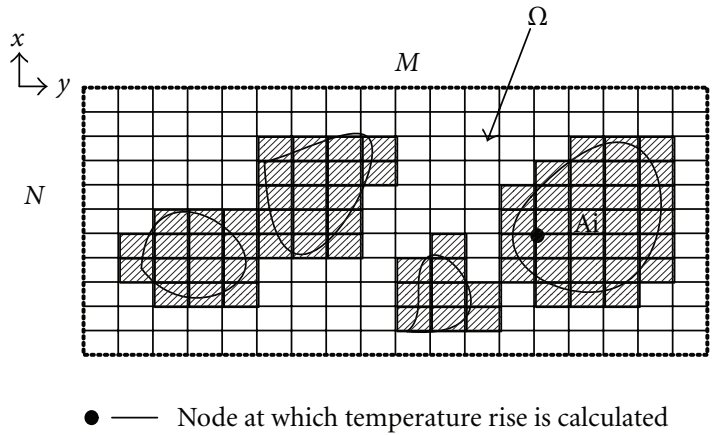

Figure 3: Grid geometry of rough surface's contact area.

The frictional heat in each node is influenced by the surrounding friction force over the entire nominal area. To calculate the temperature in just one node, the heat influence function of all the nodes must be integrated. To overcome this difficulty, the contact region $\Omega$ has been divided into $M \times N$ elements of the same size (Figure 3), so when the time of the sliding is $t$, the frictional heat matrix approach is often used:

$$
\{U\}=[T]\{Q\} \quad \text { or } \quad u=\sum_{m=0}^{M} \sum_{n=0}^{N} q_{i, j, t} \times T_{i, j, t} .
$$

$\{U\}$ and $\{Q\}$, respectively, are the frictional heat, heat flux vectors with $N$ elements and $[T]$ is the coefficient of frictional heat matrix $(M \times N)$ which is only a function of the grid geometry. $[T]$ matrix needs to be calculated first during the contact simulation. After that, the frictional heat $\{U\}$ can be obtained simply by multiplying the heat flux $\{Q\}$ with the matrix $[T]$. The frictional heat matrix method is very effective in calculating the temperature rise.

The element $T_{i, j, t}$ of the frictional heat matrix [T] could be physically interpreted as the influence factor of one unit frictional heat at node $j$ to the frictional heat at node $i$. The validity of this statement depends on the types of the interpolating functions used for the frictional heat but the overall concept is still valid. According to the laws of thermodynamics, this influence factor is only a function of the distance between the two nodes. This means that the elements of $[T]$ are determined only by the grid geometry.

The frictional heat coefficient of each element can be calculated by (9), then substitute its result to (8), and the temperature rise coefficient which was generated by the moving heat source can be worked out. Next couple it to the heat flux of every node, and finally, the temperature rise and distribution of the sliding rough surface can be obtained by (10).

2.3. Algorithm and Program. Engineering rough surface can be like a fractal phenomenon, appearing random, chaotic, self-similarity, self-affinity, and multiscale nature [8]. The fractal surface generated by square grid can be used to simulate the engineer surface efficiently. The main idea of this algorithm is that first to get a few points of the surface, then to increase their height randomly according to the normal distribution $N(0,1)$, and then incremental variance

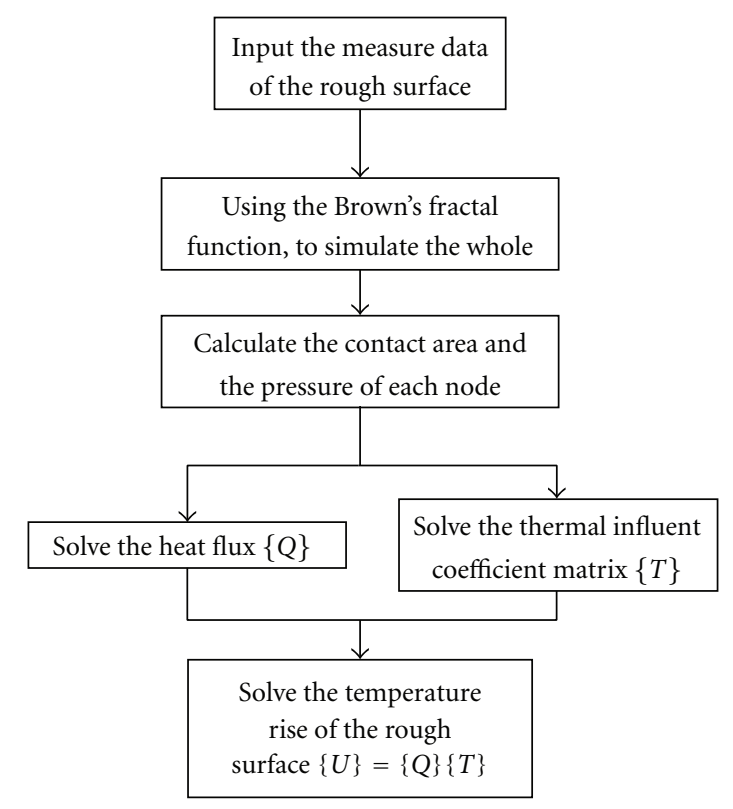

Figure 4: Overall flow chart for solving true rough surface's temperature rise.

satisfies the equation $V(t)=E[x(t)-x(0)]^{2}=|t|^{2 H} \sigma_{0}^{2}$, and the height of midpoint of time range is given by interpolation; repeat this process $n$ times continuously until the sample variance decreases to $\sigma_{n}^{2}=(1 / 2)^{2 H} \sigma_{n-1}^{2 H n} \sigma_{0}^{2}$. According to it the coordinate value of the rough surface's nodes can be obtained. And the contact area has been solved by the previous research in [10]; then the thermal influent coefficient of total nodes can be worked out; the frictional heat coefficient matrix [T] can be solved by this results easily. At the same time, the total contact area of whole patches can be obtained as well then to calculate the heat flux $\{Q\}$. The overall flow chart for the calculation is given in Figure 4 .

\section{Result and Discussion}

High-speed steel M50 was chosen as the 3D fractal profile to slide against the ceramic material $\mathrm{Si}_{3} \mathrm{~N}_{4}$ which was the semiinfinite elastic medium. Their thermal physical parameters are listed in Table 1 [11].

M50: it is a square with the length of side $0.1 \mu \mathrm{m}$. The true surface was established by using the fractal theory (Figure 5). Its roughness $\mathrm{Ra}=6.3 \mu \mathrm{m}$, the total of the nodes is $10 \times 10$; the surface of material $\mathrm{Si}_{3} \mathrm{~N}_{4}$ was seen as a relative smooth $[12,13]$.

The first calculation condition is as follows: the load $F=$ $10 \mathrm{~N}$, the sliding velocity was varied between 1 and $100 \mathrm{~m} / \mathrm{s}$. So the calculated temperature could be changed from room temperature to $1300^{\circ} \mathrm{C}$. The relationship between the sliding velocity and the temperature rise has been plotted as two curves in Figure 6.

Each curve in Figure 6 shows a significant temperature rise following the sliding velocity increase. This indicates that the sliding velocity is one of the most important factors which generate the frictional heat. 
TABLE 1: Thermal performance of materials.

\begin{tabular}{|c|c|c|c|c|c|c|c|c|}
\hline Material & Temperature $/{ }^{\circ} \mathrm{C}$ & $\begin{array}{c}\text { Young's } \\
\text { modulus/GPa }\end{array}$ & $\begin{array}{c}\text { Thermal } \\
\text { conductivity } \\
\left(\mathrm{W} \mathrm{m}^{-2}{ }^{\circ} \mathrm{C}^{-1}\right)\end{array}$ & $\begin{array}{c}\text { Expansion } \\
\text { coefficient } /{ }^{\circ} \mathrm{C}^{-1}\end{array}$ & $\begin{array}{l}\text { Specific heat } \\
\left(\mathrm{J} \mathrm{kg}^{-1}{ }^{\circ} \mathrm{C}^{-1}\right)\end{array}$ & $\begin{array}{l}\text { Friction } \\
\text { coefficient }\end{array}$ & Density $\mathrm{kg} \mathrm{m}^{-3}$ & Poisson's ratio \\
\hline \multirow{7}{*}{ M50 } & 25 & 201.76 & 43 & \multirow{7}{*}{$13.5 \times 10^{-6}$} & \multirow{7}{*}{500} & 0.60 & \multirow{7}{*}{8030} & \multirow{7}{*}{0.3} \\
\hline & 100 & 196.16 & 43 & & & 0.70 & & \\
\hline & 200 & 188.7 & 42 & & & 0.80 & & \\
\hline & 300 & 181.24 & 40 & & & 0.71 & & \\
\hline & 400 & 173.78 & 36 & & & 0.42 & & \\
\hline & 500 & 166.32 & 35 & & & 0.35 & & \\
\hline & 600 & 158.86 & 33 & & & 0.19 & & \\
\hline $\mathrm{Si}_{3} \mathrm{~N}_{4}$ & 25 & 380 & 12.6 & $3.2 \times 10^{-6}$ & 711.8 & 0.3 & 3190 & 0.25 \\
\hline
\end{tabular}

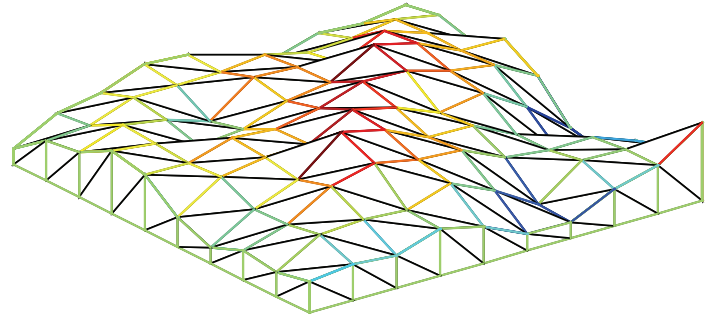

FiguRe 5: Fractal model of engineering surface.

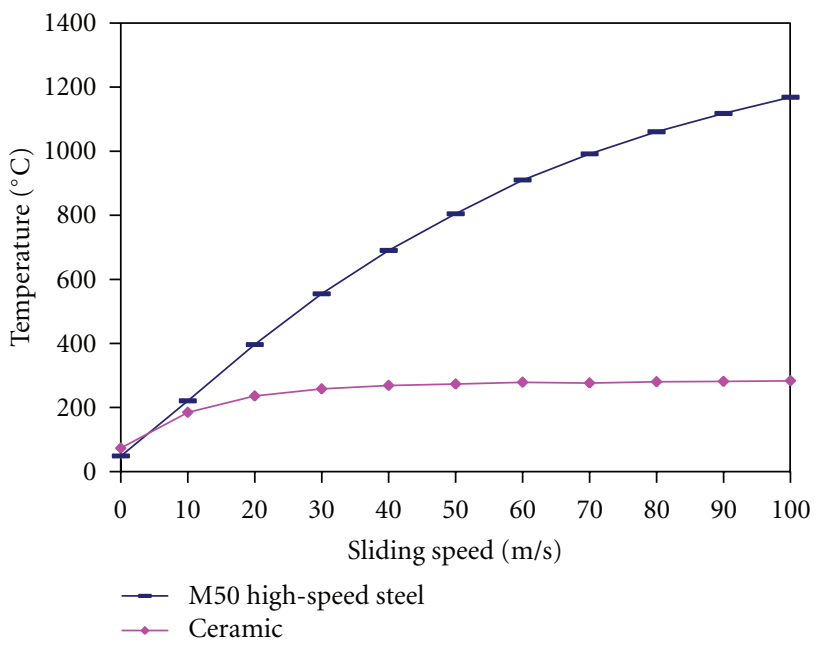

Figure 6: Effects of sliding speed on temperature rise.

The temperature of M50 is higher than the $\mathrm{Si}_{3} \mathrm{~N}_{4}$ under the same sliding velocity. And as the speed increases, the temperature rise of M50 increases faster than $\mathrm{Si}_{3} \mathrm{~N}_{4}$. The result shows that the frictional heat of the material which thermal characteristic performances better will increase less. It indicates that heat diffusion coefficient, which decides the thermal property, may be one of the key points to temperature rise.

The second calculation conditions is as follows: the sliding velocity $V=10 \mathrm{~m} / \mathrm{s}$, the load $F=10 \mathrm{~N}$, and the

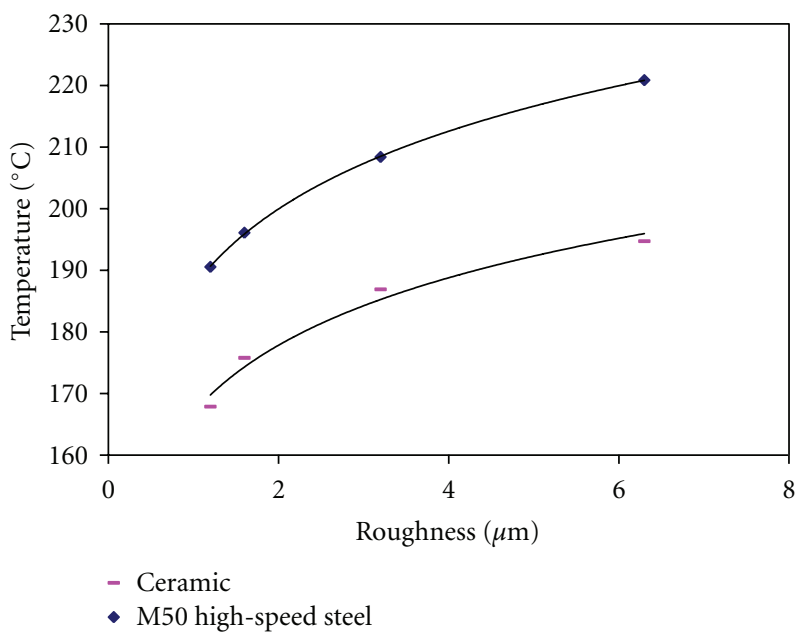

FIgURE 7: Effects of roughness on temperature rise.

roughness, respectively, was $1.2 \mu \mathrm{m}, 1.6 \mu \mathrm{m}, 3.2 \mu \mathrm{m}$, and $6.3 \mu \mathrm{m}$. So the calculated temperature could be changed from 160 to $230^{\circ} \mathrm{C}$. The relationship between the roughness and the temperature rise has been plotted as two curves in Figure 7. The result shown here reflects effects of roughness on temperature. The total contact area $\sum_{i=1}^{\text {sum }} A_{i}$, which is related to the roughness of the surface, is in proportional to the frictional heat, which tends to increase the temperature.

The third calculation condition is as follows: the sliding velocity $V=10 \mathrm{~m} / \mathrm{s}$, the roughness $\mathrm{Ra}=6.3 \mu \mathrm{m}$, and the load was varied between 10 and $100 \mathrm{~N}$. So the calculation temperature could be changed from 200 to $1800^{\circ} \mathrm{C}$. The relationship between the roughness and the temperature rise has been plotted as two curves in Figure 8. The result shown here reflects one major effect of load on temperature. The amount of frictional energy deposited on the surface is directly proportional to the load, which tends to increase temperature.

Figures 9 and 10 show a comparison temperature rise contours between M50 $(\mathrm{Ra}=6.3 \mu \mathrm{m}, F=10 \mathrm{~N}$, and $V=$ $10 \mathrm{~m} / \mathrm{s}$ ) and $\mathrm{Si}_{3} \mathrm{~N}_{4}$ (relative smooth). It can be observed that frictional heat distribution is changed by the contact area, especially that below asperity contacts in the rough surface 


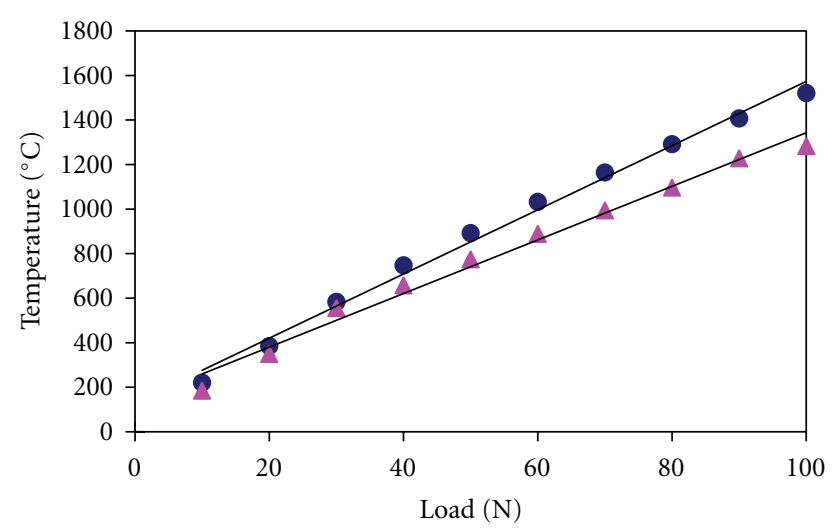

- M50 high-speed steel

$\Delta$ Ceramic

FIgURE 8: Effects of load on temperature rise.

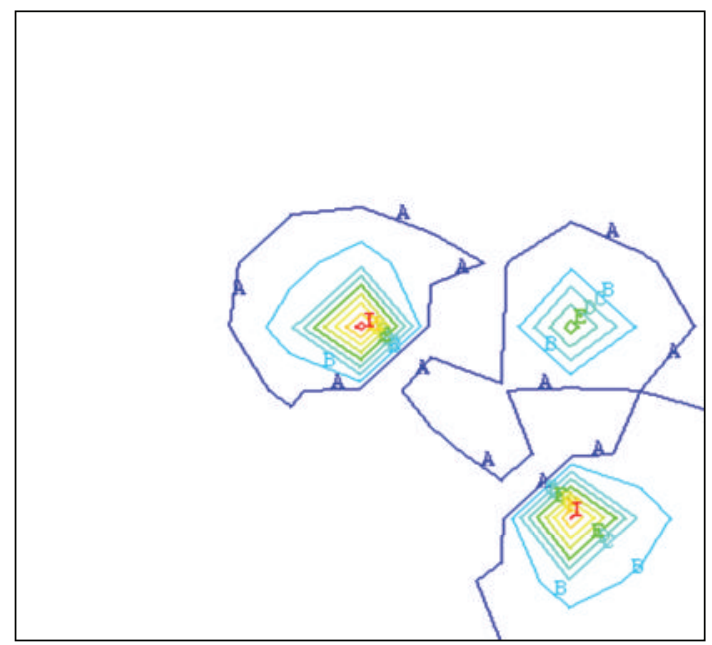

$\begin{array}{ll}\mathrm{A}=35.903 & \mathrm{~F}=144.711 \\ \mathrm{~B}=57.664 & \mathrm{G}=166.473 \\ \mathrm{C}=79.426 & \mathrm{H}=188.235 \\ \mathrm{D}=101.188 & \mathrm{I}=209.997 \\ \mathrm{E}=122.95 & \end{array}$

FIgURE 9: Temperature rise contours of M50.

where thermoelastic deformation promoted the interaction of the thermal conduct of the neighboring asperity. These analytical results are supported by FEM simulations and it shows a strong effect of the contact area on the location of the frictional heating.

Figure 11 shows a temperature rise contours of M50 on the same test condition of Figure $2(\mathrm{Ra}=3.2 \mu \mathrm{m}, F=10 \mathrm{~N}$, $T=400^{\circ} \mathrm{C}$, and $V=1 \mathrm{~m} / \mathrm{s}$ ). The result can totally interpret why there are some fusion points appearing on the M50 friction surface (the melting point of material M50 is $550^{\circ} \mathrm{C}$ ). From this method, the fusion micro-zone can be decided. It helps us to predict the fusion situation of each material's engineering surface in extreme friction condition.

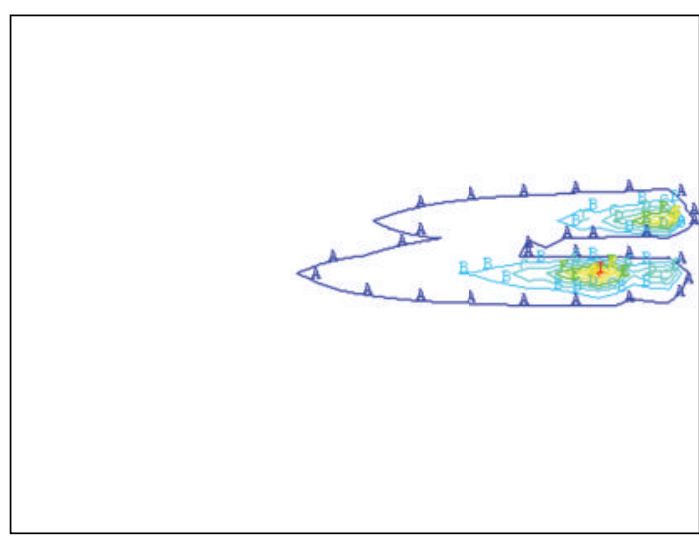
$\mathrm{A}=30.741$
$\mathrm{B}=48.858$
$\mathrm{C}=66.976$
$\mathrm{D}=85.093$
$\mathrm{E}=103.211$

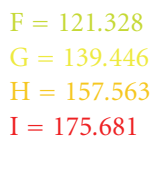

Figure 10: Temperature rise contours of $\mathrm{Si}_{3} \mathrm{~N}_{4}$.

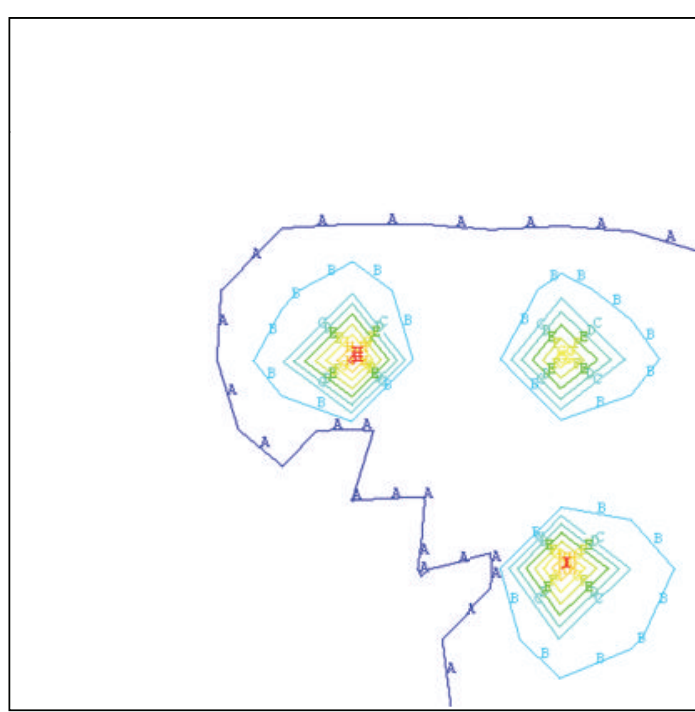
$\mathrm{A}=435.308$
$\mathrm{F}=536.827$
$\mathrm{B}=455.612$
$\mathrm{C}=475.915$
$\mathrm{D}=496.219$
$\mathrm{E}=516.523$

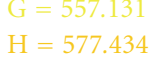
$\mathrm{I}=597.738$

FIgURE 11: Temperature rise contours of M50 on the same condition as Figure 2.

\section{Conclusions}

The focus of this paper is to deduce prediction model which can calculate the fusion micro-zone on the sliding contact surface from the viewpoint of the surface temperature rise and its distribution. It can be seen clearly that high local temperature decides the fusion zone on the engineering 
surface; the elements which contribute to the friction heat are the elements effect on the fusion zone. Case studies reveal five important trends.

(1) The maximum frictional heat is closely related to the sliding velocity, and it increases as the sliding velocity increases.

(2) The thermal properties of the material are one of the key points to temperature rise. The temperature rise decreases as the heat diffusion coefficient increases.

(3) The shape of the temperature distribution looks like an eccentric ring. The maximum hot point lies in its center.

(4) The roughness of the engineering surface has relative smaller influence on the temperature rise compared with the sliding velocity and thermal properties.

(5) The load is another main factor in increasing the temperature rise.

The important conclusion from this study is that it predicts the true engineer surface's fusion micro-zone, in the sliding process. The results may be useful for selecting material and designing the surface accuracy in the extreme working condition.

\section{Acknowledgment}

The authors would like to thank National Natural Science Foundation of China for financial support (ID 51075311).

\section{References}

[1] H. Blok, "Theoretical study of temperature rise at surfaces of actual contact under oiliness lubricating conditions," in Proceedings of the General Discussion on Lubrication and Lubricants, vol. 2, pp. 222-235, Institution of Mechanical Engineers, London, UK.

[2] J. C. Jaeger and H. S. Carslaw, Conduction of Heat in Solids, Clarendon Press, Oxford, UK, 1959.

[3] B. Vick and M. J. Furey, "A basic theoretical study of the temperature rise in sliding contact with multiple contacts," Tribology International, vol. 34, no. 12, pp. 823-829, 2001.

[4] H. Chen, W. Hu, H. Wang, and W. Wang, "Calculation of temperature fields of bodies in sliding con tact without lubrication," Journal of Tsinghua University(Science and Technology), vol. 47, no. 11, pp. 1962-1964, 2007.

[5] Q. Chen and D. Y. Li, "A computational study of frictional heating and energy conversion during sliding processes," Wear, vol. 259, no. 7-12, pp. 1382-1391, 2005.

[6] P. N. Bogdanovich and D. V. Tkachuk, "Temperature distribution over contact area and "hot spots" in rubbing solid contact," Tribology International, vol. 39, no. 11, pp. 13551360, 2006.

[7] G. Liu, Q. Wang, and Y. Ao, "Convenient formulas for modeling three-dimensional thermo-mechanical asperity contacts," Tribology International, vol. 35, no. 7, pp. 411-423, 2002.

[8] B. B. Mandelbrot, "Multifractal and fractal," Physics Today, vol. 39, no. 9, pp. 11-12, 1986.

[9] A. Majumdar and B. Bhushan, "Fractal model of elastic-plastic contact between rough surfaces," ASME-Journal of Tribology, vol. 113, no. 11, pp. 1-11, 1991.
[10] Y. Lu and Z. Liu, "Friction surface contact deformation model based onmaterial thermal parameters," Chinese Journal of Mechanical Engineering, vol. 46, no. 9, pp. 28-33, 2010.

[11] Z. Liu, "Study of critical friction and wear temperature of M50 high speed steel," Materials for Mechanical Engineering, vol. 21, no. 2, pp. 21-24, 1997.

[12] J. Yang and K. Komvopoulos, "A mechanics approach to static friction of elastic-plastic fractal surfaces," ASME-Journal of Tribology, vol. 127, no. 2, pp. 315-324, 2005.

[13] H. Sofuoglu and A. Ozer, "Thermomechanical analysis of elastoplastic medium in sliding contact with fractal surface," Tribology International, vol. 41, no. 8, pp. 783-796, 2008. 

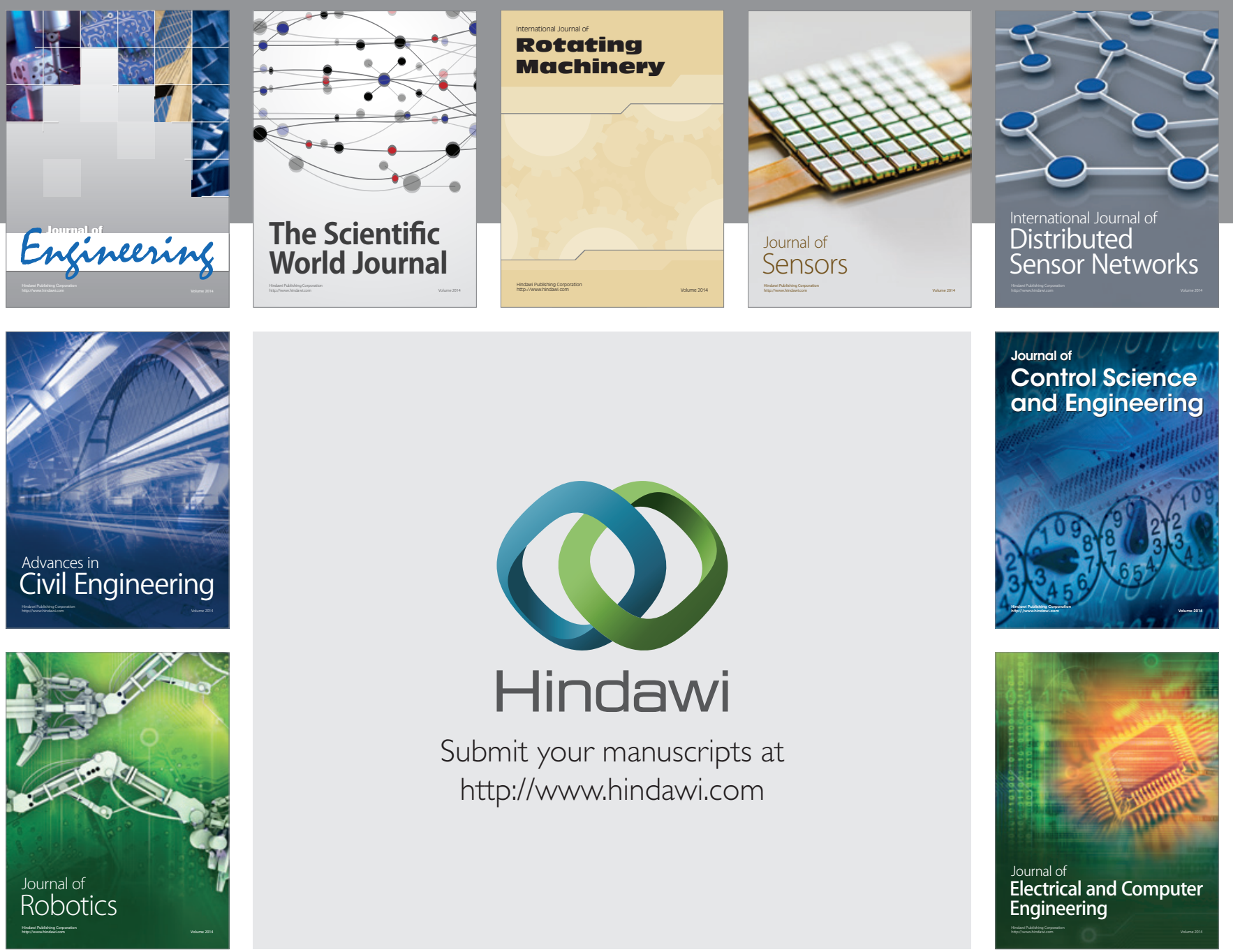

Submit your manuscripts at

http://www.hindawi.com
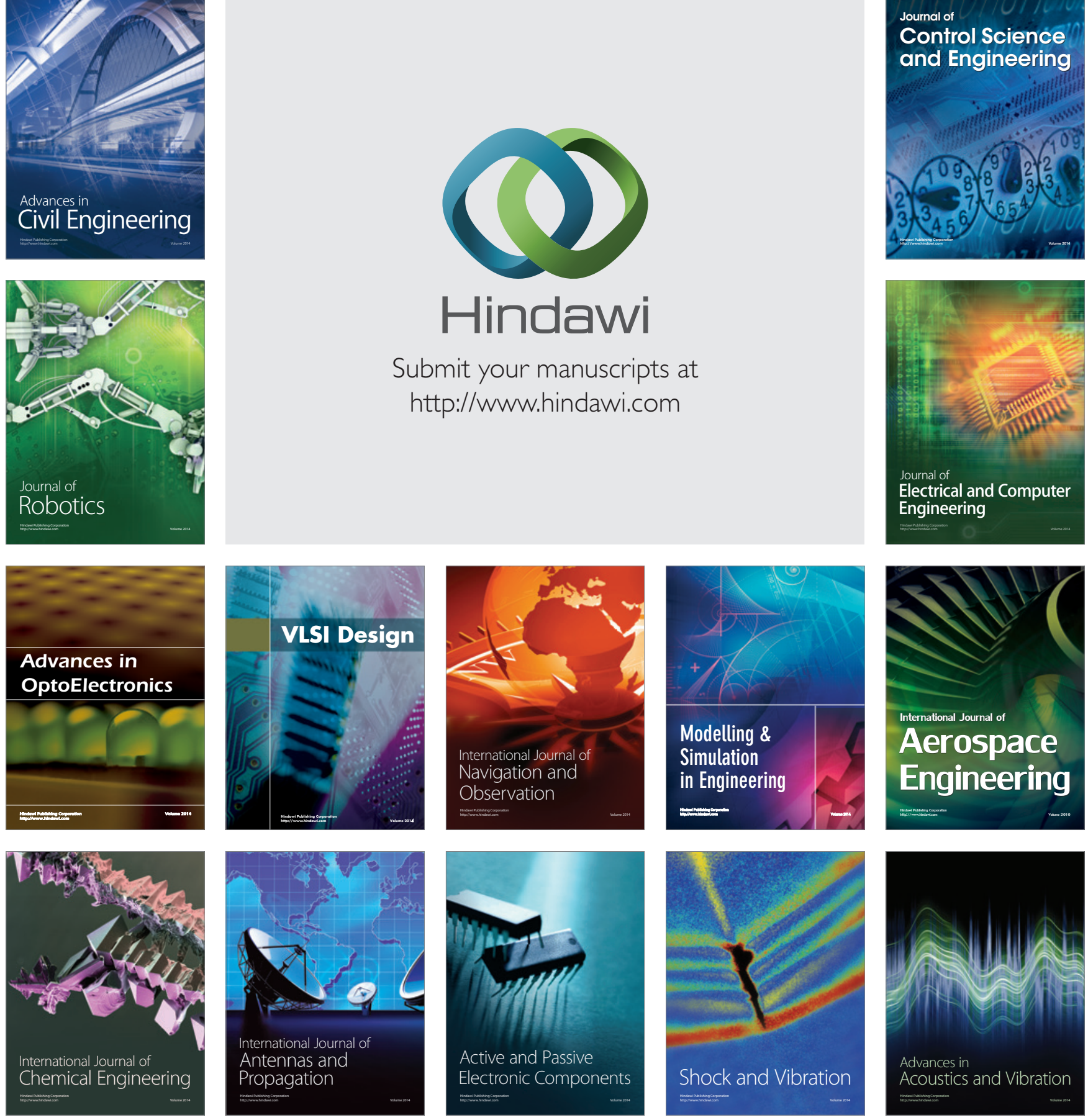